\title{
10 DEVELOPMENT AND VALIDATION OF A PARENT SURVEY FOR REPORTING CHILD INJURIES
}

doi:10.1136/injuryprev-2012-040590w.10

${ }^{1} \mathrm{~A}$. Curry, ${ }^{1,2} \mathrm{M}$ Zonfrillo, ${ }^{1} \mathrm{R}$ Myers, ${ }^{1,2} \mathrm{D}$ Durbin. ${ }^{1}$ Center for Injury Research and Prevention, The Children's Hospital of Philadelphia, Pennsylvania, USA; ${ }^{2}$ Department of Pediatrics, Perelman School of Medicine, University of Pennsylvania, USA

Background Medical record abstraction is considered the 'gold standard' method of ascertaining injury information, but can be time-consuming and costly.

Aims/Objectives/Purpose To assess the ability of parents to identify (1) moderate and greater severity injuries and (2) serious injuries, overall and by body region, by comparing survey responses to hospital medical records.

Methods Children $\leq 17$ years treated for injuries at three emergency departments between $08 / 10$ and $07 / 11$ and their parents were eligible. Parents were randomised to either a telephone interview or self-administered survey at 2 weeks post-visit and asked to report child injuries to seven body regions. The Abbreviated Injury Scale (AIS) score was used to assign each child a body-region injury severity level of minor, moderate, or serious, generally corresponding to AIS scores of 1,2 and $>3$.

Results/Outcomes Of 798 parents enrolled in the study, 494 (61.9\%) completed the survey. The overall sensitivity, reflecting the ability of parents to identify a documented moderate or serious injury, was 0.94 (95\% CI 0.93 to 0.97 ). Sensitivities were $>0.90$ for the face and upper and lower extremity regions, $0.80-0.90$ for head, abdomen, neck/back/spine regions, and $<0.60$ for the thorax region. Specificities for all body regions were $\geq 0.95$. The overall sensitivity for identification of a serious injury (AIS $>3$ ) was 0.71 $(0.60,0.80)$.

Significance/Contribution to the Field This survey enables parents to identify and characterise the body region and severity of their child's injuries and to differentiate between minor and more significant injuries. This survey may be particularly valuable in population-based studies of paediatric injuries. 